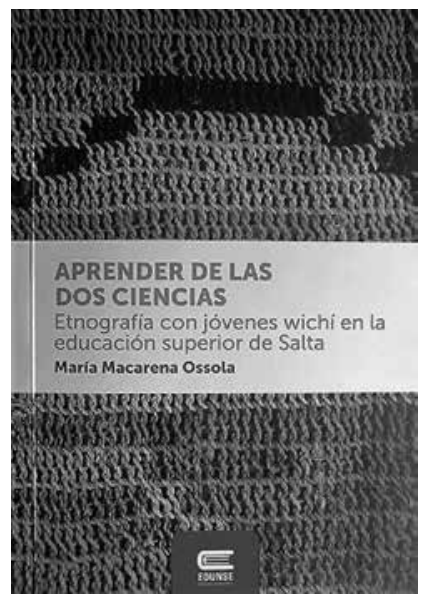

Young Wichi University Students

GLORIA MANCINELLI Facultad de Filosofía y Letras, Universidad de Buenos Aires,

Buenos Aires, Argentina mancinelli.gloria@gmail.com

Desacatos 55 ,

septiembre-diciembre 2017, pp. 202-207
- Aprender de las dos ciencias.

Etnografía con jóvenes wichi en

la educación superior de Salta

María Macarena OSSOLA, 2015

Editorial de la Universidad Nacional de

Santiago del Estero, Santiago del Estero

\section{Jóvenes, universitarios y wichí}

\author{
GLORIA MANCINELLI
}

$A$

prender de las dos ciencias puede considerarse uno de los primeros trabajos etnográficos en Argentina sobre la experiencia de los jóvenes indígenas al transitar por su formación superior universitaria en espacios académicos tradicionales. Emplazada en la Universidad Nacional de Salta, esta investigación logra dar testimonio de las complejas aristas que reviste la inclusión de jóvenes indígenas en el nivel superior. Al mismo tiempo, hace visible un campo aún poco explorado en nuestro país en las investigaciones referidas a procesos educativos, en particular aquellos que se enfocan en espacios formales. El trabajo de Ossola se inserta en un área considerada en vacancia no sólo por la escasa producción de investigaciones realizadas en ese campo, sino también porque el derecho a acceder a formación superior se constituye hoy como una de las principales demandas de los colectivos indígenas tanto de Argentina como de otros países de Latinoamérica. La relevancia de este trabajo puede entenderse también si se considera que los jóvenes que configuran esta etnografía pertenecen a una de las poblaciones con las tasas más bajas de acceso a educación en todos los niveles de enseñanza, entre los que el analfabetismo sigue siendo uno de los principales problemas y pocos culminan el nivel medio, requisito necesario para ingresar al nivel superior - terciario y 
universitario-. Cabe aclarar que la provincia de Salta, donde se sitúa la experiencia de este trabajo, es una de las que reúne la mayor diversidad étnica, con pueblos como wichí, tupi-guaraní, chané, tapieté, toba, chorote, chulupí, kolla y diaguita-calchaquí. En conjunto, estas poblaciones comparten problemas educativos similares y contextos de profunda desigualdad social en el acceso a recursos básicos, como el trabajo, la educación, la salud y el territorio (Trinchero, 2000; Buliubuasich, 2009).

No obstante, puede decirse que la Universidad Nacional de Salta (UNSa) comienza a asumir la presencia de indígenas en sus espacios. La "presencia" o "ausencia" no debe entenderse sólo como estar o no en la universidad, sino más con el modo de estar “presente para la institución” (p. 96).

La investigación con la que comienza este trabajo se desarrolla en el marco de la formación doctoral de Ossola, antropóloga social por la UNSa, en el área de antropología social y cultural de la Facultad de Filosofía y Letras de la Universidad de Buenos Aires, con beca del Consejo Nacional de Investigaciones Científicas y Técnicas. El proceso fue acompañado por Catalina Buliubasich, Carolina Hecht y Gabriela Novaro, referentes en el campo de las investigaciones centradas en pueblos indígenas y educación intercultural bilingüe. La reelaboración de su tesis doctoral, Jóvenes wichí en la educación superior de Salta: interpelaciones escolares, étnicas y etarias, da origen a este texto del que me interesa recuperar las principales interrogantes que Ossola aporta como líneas de indagación y problematización para continuar profundizando en el área.

Coincido con Mariana Paladino (2009) ${ }^{1}$ en que uno de los aspectos clave de este libro es entender la presencia indígenas en la universidad, no ya como objeto de estudio. Por el contrario, el texto describe los procesos de apropiación y producción de espacios en la UNSa por parte de jóvenes universitarios wichí (JUW), ${ }^{2}$ los comprende en su condición de sujetos y su capacidad de agencia. De acuerdo con esto, la producción cultural de los JUW, en tanto “indígenas universitarios” en la universidad y "jóvenes indígenas" en la comunidad (p. 234), a partir de su experiencia en la educación superior es el tema central del trabajo. El objetivo es exponer la capacidad de los JUW para interpelar las perspectivas de actores y espacios institucionales, tanto en la UNSa como en la comunidad a la que pertenecen.

Ossola eligió una metodología cualitativa, basada en entrevistas en profundidad que permitieron una elaboración detallada de las trayectorias escolares de los JUW para ingresar a la universidad. El trabajo de campo bisituado ayudó a construir un registro del impacto de esta experiencia dentro de la universidad en el contexto de la comunidad wichí a la que pertenecen los JuW. ${ }^{3}$

El marco teórico destaca por la recuperación de trabajos propios del campo de la antropología educativa, la sociología de la educación y el campo de la lingüística. Incorpora también autores cuyos trabajos se han enfocado más en la variable étnica y los procesos económicos, sociales, culturales y políticos que sitúan al pueblo wichí en el presente, como una formación social e histórica (Trinchero, 2000).

Al ubicar a la población wichí en el espacio y el tiempo, el trabajo logra expresar los sentidos y las representaciones que la educación formal — “escuela”, "universidad"- - adquiere en estos contextos rurales,

Doctora en antropología por la Universidad Federal de Río de Janeiro, profesora adjunta en el área antropología y educación de la Facultad de Educación en la Universidad Federal Fluminense. Sus principales líneas de investigación son antropología y educación, educación superior indígena y juventud indígena.

2 Jóvenes universitarios wichí (Juw) es una categoría analítica conceptual elaborada por la autora de la tesis en el proceso de investigación.

3 La comunidad Los Troncos se ubica al noreste de la provincia de Salta, en el departamento de Rivadavia. Tanto el nombre de la comunidad como el nombre de los estudiantes son ficticios para resguardar su confidencialidad. 
interculturales e interétnicos. Sobresalen los procesos de apropiación y negociación de las comunidades en estos espacios institucionales.

En este marco se presentan las trayectorias escolares de los JUW, que nos dejan asomarnos a las vivencias internalizadas de sus experiencias escolares, que involucran los acontecimientos significativos, como situaciones de encuentro, desencuentro y tensiones con la lengua hegemónica —el españoly una experiencia interétnica particular circunscrita al entorno escolar. En estas trayectorias puede verse cómo estos jóvenes serán los primeros de la comunidad en proyectar como horizonte la asistencia a una universidad a partir del contacto que se produce entre ellos y un grupo de investigación-acción de la UNSa. Esta experiencia puede leerse no sólo como un hito, sino también como una instancia de reflexión para quienes nos abocamos a la investigación en temas de inclusión educativa. El texto aporta el dato de que estos estudiantes desconocían por completo la existencia de las universidades, pese a que eran estudiantes avanzados en el nivel medio. Si bien el texto no profundiza en este punto, al recuperarlo como acontecimiento, convoca a una serie de interrogantes sobre los horizontes pedagógicos y didácticos que tienen los docentes de escuelas medias en estas poblaciones: ¿qué percepciones tienen los docentes sobre las posibilidades de continuidad en los niveles terciario y universitario para los jóvenes indígenas que estudian en estas escuelas? ¿A partir de qué se construyen estas percepciones? ¿De qué modo influyen en las posibilidades de acceso al nivel superior en estas poblaciones?

El texto muestra que la presencia de la universidad en el espacio escolar de la comunidad permitió proyectar la posibilidad de asistir como un nuevo horizonte, a la vez que conformó un desafío etario y generacional. A partir de la ayuda de particulares y la expansión progresiva de las redes de apoyo durante el proceso de ingreso y permanencia en la universidad - acompañamiento de particulares y pares, acceso a becas de estudio, refrigerio y alojamiento-, los JUW fueron sorteando las limitaciones económicas y las necesidades de apoyo socioafectivo para ingresar y permanecer en la universidad.

Como se describe al comienzo de la reseña, Salta es una provincia con gran diversidad étnica y lingüística, combinada con una profunda desigualdad social, económica, política y cultural. Si bien los contextos de desigualdad social y económica son muy marcados en la población indígena, alcanzan también a poblaciones criollas ${ }^{4}$ rurales y urbanas.

Con esto en mente, el texto acierta en recuperar la trayectoria histórica de la UNSa, que se caracteriza por que la mayoría de sus alumnos representa la primera generación dentro de sus familias que ingresa a la universidad y porque se expresa una marcada heterogeneidad en el rendimiento académico, producto de trayectorias escolares diversas. Así, una problematización que este libro recupera se refiere a las políticas de inclusión educativa que ponderan la variable étnica. Esta perspectiva permite pensar de qué modo los imaginarios acerca de "la universidad" deben problematizarse al momento de registrar estas experiencias en las que se instituyen nuevos vínculos entre universidades y pueblos indígenas.

El texto profundiza en el entramado institucional que da forma a una de las primeras políticas que se producen en la institución para atender la inclusión de jóvenes indígenas: el Programa de Tutorías para Estudiantes de Pueblos Originarios (Protepo).

Ossola plantea cuestiones que me parecen centrales: ¿cuáles son los criterios de clasificación del “otro cultural”? ¿Qué acciones específicas supone su acompañamiento pedagógico? Estas preguntas

$4 \quad$ El término criollo tiene su origen en el periodo colonial. Aludía a personas de origen europeo. Hoy se utiliza sobre todo en el norte del país para referirse a personas no indígenas descendientes de inmigrantes. 
confrontan otras no menos dilemáticas: ¿qué resulta más adecuado: implementar políticas basadas en la desigualdad económica o en la diversidad cultural? ¿Qué implica ponderar una variable sobre la otra? Si bien estas interrogantes representan los debates más frecuentes en relación con políticas de educación intercultural y de inclusión educativa, lo relevante en este caso es el modo en que se plasman en el nivel superior, pues estas inquietudes se han manifestado principalmente, hasta ahora, sólo en los niveles inicial y primario.

Aunque el libro no pretende responder a estas preguntas, logra mostrar la complejidad del contexto en el que estas discusiones adquieren sentido y vigencia, y contribuye a los debates respecto a la producción de políticas orientadas a atender la inclusión, la igualdad educativa y el modo en que la educación superior, concebida desde una perspectiva universalista, entra en tensión con las nuevas demandas.

En términos generales, la etnografía se enfoca en las maneras en que la agencia de los "jóvenes" "universitarios" "indígenas" confronta los parámetros de clasificación del ser indígena por parte de la institución, promueve debates y orienta modificaciones en los términos de dichas clasificaciones. De aquí que las preguntas mencionadas se tornen más complejas al dejar entrever la heterogeneidad de los grupos étnicos que conforman el conjunto construido "pueblos indígenas". Este aspecto se desarrolla en el relato del ingreso de estudiantes kolla, ${ }^{5}$ un año después de que entraron los JUW, y muestra fuertes limitaciones en el diseño del Protepo. En mi opinión, la cuestión de la heterogeneidad es uno de los aportes más ricos del trabajo, ya que puede verse que la tendencia a homogeneizar el conjunto "ser indígena" en la institución, los docentes y quienes configuran políticas educativas desestiman otras variables, como la edad, la cual toma fuerza en esta etnografía.

La experiencia universitaria de los jóvenes wichí describe la manera en que se instituyen formas de ser universitario. Al recuperar las rutinas, las interacciones áulicas, la socialización entre estudiantes, la organización del tiempo y el uso de la lengua wichí como elementos para la indagación, puede verse la profusión de aspectos que intervienen desde una perspectiva etnográfica orientada a recuperar las vivencias y los procesos subjetivos de los estudiantes indígenas. Asimismo, se muestra de qué manera las propias trayectorias escolares son repensadas y la manera en que se resignifican los modos de percibir la pertenencia étnica. Así, "la identidad", lejos de asumirse como algo dado, se comprende en un proceso permanente de producción, apropiación y reconfiguración en torno de las experiencias vividas por los sujetos.

El modo en que los aprendizajes realizados en la escuela antes del ingreso a la universidad - en especial, los que no fueron adquiridos - son revisados y cuestionados por los mismos estudiantes durante la experiencia universitaria permite reflexionar sobre las decisiones didácticas y pedagógicas, pero también respecto a las políticas educativas. Desde este lugar, pueden retomarse las interrogantes planteadas arriba acerca de los horizontes escolares que asumen los docentes en espacios rurales, interculturales y bilingüies: ¿cuáles son las decisiones pedagógicas y didácticas que se toman y cómo en estas decisiones se pone o no en juego la variable étnica? ¿Las miradas, los cuestionamientos y las críticas que los JUW hacen pueden orientarnos en la producción de respuestas a los problemas de desigualdad educativa?

Lo cierto es que la falta de articulación entre la secundaria y la universidad es un problema que se expresa en muchas otras poblaciones y representa

Se compara el proceso de incorporación de estudiantes kolla, que presenta diferencias respecto al ingreso de los wichí en la selección y el perfil de los estudiantes. Esta comparación enriquece el trabajo, pues permite reflexionar sobre cómo la perspectiva de las políticas de inclusión confrontan la heterogeneidad inherente a todo proceso social. 
mayores dificultades en contextos de desigualdad profunda en el acceso a bienes y servicios. En muchas poblaciones, la desigualdad económica se imbrica con procesos ligados a la discriminación, el racismo, la folclorización y la cosificación cultural. En el caso de los grupos étnicamente definidos, estos procesos parecen adquirir sus propias particularidades y se suman al hecho de que las lenguas maternas aprendidas en el seno familiar y apropiadas por las nuevas generaciones en múltiples contextos de aprendizajes y de sociabilización se siguen concibiendo de modo subalterno (Buliubasich, 2009; Hecht, 2014).

Desde la perspectiva bisituada para el trabajo de campo y el énfasis en recuperar la agencia de los sujetos, la etnografía acompaña el retorno de los JUW a la comunidad. El capítulo cinco indaga en algunos procesos de reconfiguración social y etaria que se producen en la comunidad en el marco de esta experiencia. En este capítulo, es interesante ver cómo se amplía la información referida a la organización social y política de la población wichí, en particular de la comunidad de Los Troncos, las cual se ha transformado a lo largo de múltiples procesos políticos, económicos, sociales e históricos. De acuerdo con esto, Ossola se enfoca en dos figuras centrales que permiten comprender dinámicas clave de la organización política en las comunidades wichí: los ancianos y los caciques. Según el texto, en términos generales, los ancianos han manifestado respeto y reconocimiento al esfuerzo realizado por los jóvenes universitarios, insisten en recordar que "la educación ha estado presente desde siempre en la población wichí” (p. 197), y que su papel como ancianos es acompañar de igual manera al conjunto de los jóvenes de la comunidad más allá de sus trayectorias escolares. Resulta interesante comprender de qué modo una institución como el "consejo de ancianos", lejos de mostrarse cosificada en el tiempo y desconocida por las nuevas generaciones, es objeto de reflexión y de nuevas formas de apropiación, y presenta plena vigencia como institución ordenadora de la vida social en las comunidades wichí. Asimismo, la idea de la presencia de la educación en el pueblo permite confrontar los múltiples sentidos que cobra el concepto de educación y la manera en que se entrelazan en estos contextos con la educación formal.

En el caso de los caciques, el vínculo con los JUW se torna más complejo y el texto trata con mucho cuidado los momentos clave de esta complejidad. Puede decirse que en las comunidades indígenas el acceso a bienes y servicios va de la mano con la configuración de espacios de negociación con instituciones del Estado, en la medida en que se tejen y amplían redes sociales con particulares, con organizaciones políticas y no gubernamentales (Ossola, 2015; Mancinelli, 2014). La capacidad de sostener y expandir estos vínculos para garantizar el acceso, aunque precario, a bienes y servicios, otorga estatus social dentro de la comunidad y configura liderazgos. El texto muestra que no sólo los nuevos conocimientos adquiridos en la universidad ponen en tensión los vínculos entre estos jóvenes y los caciques, sino también las redes que estos jóvenes generan durante su experiencia universitaria. Con esta perspectiva, Aprender de las dos ciencias logra dimensionar los complejos sentidos y representaciones que la formación universitaria adquiere en estos contextos rurales, interculturales, bilingües, traslapados con escenarios de profunda desigualdad social, económica, política y cultural. D 


\section{Bibliografía}

Buliubasich, Catalina, 2009 "Derechos indígenas: la agenda urgente”, en Info-UNsa, núm. 2, p. 11.

Hecht, Ana C., 2014, "Escolarización de hablantes toba/qom: cruces entre políticas, estadísticas y trayectorias escolares", en Papeles de Trabajo, Centro de Estudios Interdisciplinarios en Etnolingüística y Antropología Social, núm. 27, pp. 103-127.

Mancinelli, Gloria, 2014, "Sentidos y perspectivas en las demandas de educación superior en las comunidades wichí. Pensando antecedentes y trayectorias históricas”, ponencia presentada en el XI Congreso Argentino de Antropología Social, Rosario, 23 al 26 de julio.

Ossola, María Macarena, 2015 Aprender de las dos ciencias. Etnografía con jóvenes wichí en la educación superior de Salta, Editorial de la Universidad Nacional de Santiago del Estero, Santiago del Estero.

Paladino, Mariana, 2009, "Pueblos indígenas y educación superior en la Argentina. Datos para el debate", en Inclusión Social y Equidad en la Educación Superior, núm. 6, pp. 81-122.

Trinchero, Héctor Hugo, 2000, Los dominios del demonio, Editorial Universitaria de Buenos Aires, Buenos Aires. 